\title{
El potencial analitico de las Redes socio-métricas y ego-centradas: una aplicación al estudio de la Cohesión-Integración de Colectivos sociales ${ }^{1}$
}

\author{
The analytical potential of social-metric and self-centered \\ Networks: an application to the study of Cohesion-Integration \\ of the social Collectives
}

\author{
Carlos lozares, Joan Miquel Verd, Oriol Barranco \\ Universidad Autónoma de Barcelona \\ carlos.lozares@uab.cat (ESPAÑA)
}

Recibido: 14.01 .2012

Aceptado: 21.03.2013

\section{RESUMEN}

Este artículo vincula diferentes campos teórico-conceptuales, perspectivas metodológicas y métodos habitualmente yuxtapuestos en el quehacer sociológico. Desde el punto de vista teórico se centra en las ideas de Cohesión e Integración como expresiones de la distribución de los recursos en Capital Social. Desde una perspectiva entre conceptual y metodológica se hace referencia a la teoría y análisis de las Redes sociales y a conceptos también intermediarios entre Redes, Cohesión, Integración y Capital social como son los de Bonding y Bridging. La referencia empírica de su aplicación son seis colectivos, expresión de modos diferentes de pertenencia e incorporación a la sociedad. En el terreno del análisis e interpretación se recurre, por un lado, a la combinación de conceptos expresados en variables clásicas atributivas y, por otro, a variables relacionales bajo dos modos, las socio-métricas y ego-centradas. En este sentido, se trata también de mostrar la eficacia y pertinencia de las redes socio-métricas y egocentradas y la de sus indicadores.

${ }^{1}$ El análisis de este artículo se hace a partir del marco teórico y datos de la investigación CASREDIN (Estudio comparado de casos sobre la influencia mutua entre capital e integración sociales y la inserción, estabilidad, promoción y cualificación en el empleo), proyecto I+D+I con referencia CSO2008-01470, subvencionado por el Ministerio de Educación y Ciencia dentro del marco del VI Plan Nacional de Investigación Científica, Desarrollo e Innovación Tecnológica 2008-2011.

EMPIRIA. Revista de Metodología de Ciencias Sociales. N. ${ }^{\circ}$ 26, julio-diciembre, 2013, pp. 35-62. ISSN: 1139-5737, DOI: 10.5944/empiria.26.7152 


\title{
PALABRAS CLAVE
}

Cohesión, Integración, Capital social, redes sociométricas, redes ego-centradas.

\begin{abstract}
This article links different theoretical-conceptual fields, methodological perspectives and methods usually juxtaposed in sociological work. From a theoretical point of view it focuses on the idea of cohesion and integration as expressions of the distribution of resources of Social Capital. In a perspective between conceptual and methodological issues it refers to the theory and analysis of social networks and also to intermediate concepts between networks, cohesion, integration and social capital such as Bonding and Bridging. The empirical references of its application are six human collectives, which are the expression of different modes of belonging and integration into society. In terms of analysis and interpretation, on the one hand, a combination of classical socialdemographical variables is used, and, on the other hand, relational variables are used. These latter variables are expressed under two modes, socio-metric and ego-centred. In this sense the article also shows the effectiveness and relevance of the socio-metric and ego-centred networks and its indicators.
\end{abstract}

\section{KEY WORDS}

Cohesion, integration, social capital, sociometric networks, ego-centered networks.

\section{INTRODUCCIÓN}

Este artículo tiene tres objetivos articulados entre sí. El primero, consiste en identificar y operativizar los conceptos de Cohesión e Integración social desde dos perspectivas. La primera, que denominamos Cohesión-Integración relacional, se basa en identificar dichos conceptos exclusivamente a partir de las relaciones entre los agentes de un colectivo previamente definido; la segunda, que denominamos relacional-atributiva, consiste en identificarlos a partir de la perspectiva relacional precedente pero haciendo intervenir los atributos sociales de los agentes para caracterizar los estatus o posiciones de la estructura relacional previa. Estos dos modos de entender los conceptos de Cohesión-Integración se enmarcan en el dilema con que pueden concebirse los hechos o fenómenos sociales: de un lado, partir de las interacciones y relaciones como generadoras de la estructura reticular y social, más propio a la idea de Cohesión-Integración; de otro lado, partir de las características o atributos sociales previos para identificar en ellos sus características relacionales. 
El segundo objetivo del artículo es metodológico, basado en la combinación de redes socio-métricas y ego-centradas. Este objetivo está vinculado al precedente, pues se trata de mostrar el potencial del análisis de Redes sociales, de sus conceptos e indicadores para dar cuenta de las dos versiones anunciadas de la Cohesión-Integración. En primer lugar, la redes sociométricas, en perspectiva relacional, conllevan una distribución y clasificación de sus relaciones y agentes por la estructura de relaciones que generan. Si además se consideran los atributos socio-demográficos u otros de los agentes, es posible verificar el correlato entre la estructura de redes y la de los atributos. Es lo que se hace en la que denominamos la versión relacional-atributiva de las redes sociométricas. En segundo lugar, las redes ego-centradas parten de que cada Ego está relacionado con todos sus Alteri y, a su vez, los Alteri entre sí, sin que necesariamente exista relaciones entre los Egos. En una versión estrictamente relacional de las redes ego-centradas, el análisis consiste en estructurar las relaciones o redes socio-métricas de los Alteri por criterios reticulares de Cohesión-Integración, tal y como serán definidos, atribuyendo tales propiedades estructurales a cada Ego y posibilitando su clasificación. Si adicionalmente se consideran los atributos de los Egos, es factible poner en correspondencia las estructuras relacionales de los Alteri atribuyendo las estructuras de los Alteri a las categorías de los Egos. Es la versión relacional-atributiva en redes egocentradas.

El tercer objetivo del artículo consiste en comprobar la pertinencia de los dos tipos de Cohesión-Integración, tanto en las redes socio-métricas como en las ego-centradas, por medio de los indicadores y métodos de las redes sociales. Para ello, el análisis se lleva a cabo en un caso concreto analizando la CohesiónIntegración de seis colectivos en que intervienen atributos vinculados a la edad y origen de una población. Como hipótesis genérica no se trata tanto de validar unos contenidos sustantivos, que también, como de mostrar la bondad de una metodología específica para identificar los dos tipos de Cohesión-Integración, relacional y relacional-atributiva, en redes socio-métricas y ego-centradas por medio de una aplicación sustantiva a un caso específico. En este sentido, la teoría del capital social, de la que no se hace un desarrollo exhaustivo, es la orientación teórica y conceptual que fundamenta y enmarca tanto los conceptos de Cohesión-Integración, como sus formas más operativas en Bonding y Bridging/ Linking, así como la perspectiva relacional.

Los tres objetivos y las temáticas señaladas se desarrollan en sucesivos apartados. En el primero, se explican las concepciones de estructura relacional, Cohesión e Integración social y capital social. En el segundo, se exponen las características de las dos versiones o modalidades de Cohesión-Integración relacional y relacional-atributiva. En el tercer apartado se identifican los colectivos que son objeto de estudio, las características de la encuesta y de los datos en que se basa el artículo. En el cuarto apartado se aplican y analizan las diferentes modalidades de Cohesión-Integración en los colectivos estudiados por medio de redes socio-métricas, ego-centradas y su combinación. Por último, en el quinto apartado, se exponen las conclusiones del artículo.

EMPIRIA. Revista de Metodología de Ciencias Sociales. N. ${ }^{\circ}$ 26, julio-diciembre, 2013, pp. 35-62. ISSN: 1139-5737, DOI: 10.5944/empiria.26.7152 


\section{LAS RELACIONES BONDING Y BRIDGING/LINKING COMO MODOS DE ABORDAR EL CAPITAL SOCIAL DE UN COLECTIVO Y/O ENTRE COLECTIVOS DIFERENTES}

\subsection{Bonding, Capital social y Cohesión en un colectivo}

\section{Bonding y Capital social}

Las relaciones o interacciones Bonding de un colectivo, grupo, asociación o población se definen como los vínculos hacia dentro o entre los agentes de dicho colectivo (Borgatti, Jones y Everett, 1998). Esta configuración de las relaciones constituye la estructuración reticular interna del colectivo (Atria, 2003). El número, frecuencia y densidad de los Bonding es variable. Los Bonding son relaciones de tipo horizontal en el sentido de que son vínculos entre agentes que son equivalentes socialmente (Adler y Kwon, 2002).

Los contenidos de las relaciones pueden ser diversos, por ejemplo de naturaleza económica, informativa de tipo instrumental, de apoyo mutuo y/o de confianza, unas objetivadas y otras cognitivas. Los Bonding con contenidos de normas comunes, confianza y apoyo mutuo, denominados a veces como relaciones fuertes, son expresiones de capital social que consolidan o introducen coordinación y cooperación en los colectivos o en las instituciones democráticas. De dicho Capital social, en tanto que recurso interno de los colectivos, se benefician también los agentes individuales (Coleman, 1998; Putnam, 1993; 1995; Portes, 1998; Bourdieu, 1986; Pöyhönen, 2005; Lin 1999; 2001)²

\section{Cohesión social y Capital Social}

La Cohesión social no es de simple y homogénea definición (Levi, 1998) 3 . De la revisión de sus definiciones se concluye que se trata de un concepto que

\footnotetext{
2 Desde un punto de vista más específico, para relaciones entre Capital-Integración social, Bonding-Linking/Bridging, Cohesión-Integración ver también Lozares, Verd, López-Roldán, Martí y Molina (2011); Lozares, López-Roldán, Verd, Martí, Molina, Bolibar y Cruz (2011); y Lozares y Verd (2011).

${ }_{3}^{3}$ Woolley (1998) la considera como valores participados e interacción social y ausencia de exclusión; Jenson (1998) como inclusión, participación, reconocimiento y legitimidad; Forrest y Kearns (2001) como valores y objetivos comunes, solidaridad social, redes y sentido de atracción e identidad vinculadas a un lugar o grupo; O'Reilly et al. (1977) como desarrollo de la conectividad; Festinger et al. (1950) como un campo de fuerzas para que los miembros permanezcan en grupo; Gross Martin (1952) como resistencia a fuerzas disruptivas de un colectivo; Beck, van der Maesen et al. (1997) como proceso que genera redes sociales e infraestructuras que las sostengan para que los ciudadanos existan como sujetos reales humanos y sociales; para Luhmann (1988) se trata de la confianza necesaria para arriesgarse y tanto más cuanto más lo hagan otros miembros del colectivo. Beck (1997) considera la Cohesión social como el pegamento que vincula la sociedad como conjunto, la solidaridad y las relaciones sociales, los valores e identidades: sin la Cohesión social no hay interacción y sin ella no hay identidad colectiva y vida social. Para Jeannotte (2003) la Cohesión social corresponde a la idea de pertenencia, inclusión, participación, reconocimiento y legitimidad. Bernard (1999) lo relaciona con la idea de igualdad (ver también Lozares, López-Roldán, Verd, Martí, Molina, Bolibar y Cruz, 2011; Lozares y Verd, 2011; Lozares, 1996; Lozares, 2006).
} 
se asocia a identidad, inclusión, igualdad, solidaridad, homogeneidad, uniformidad, confianza, valores compartidos, cultura propia, participación, etc. dentro de un colectivo. Además puede hablarse de sus grados de intensidad (Moody y White, 2003). Los contenidos pueden ser culturales, educativos, político-ciudadanos, económicos, etc. (Amin, 2002; Cheong et al., 2007) y las políticas para su implementación diversas (Baker et al., 1999; Schuster y Solomos, 2004; Amin, 2002; Arneil, 2006; Ben-Tovim, 2002). Es decir que la polisemia está servida.

Por su parte, la revisión de las muchas definiciones existentes de capital social pone de manifiesto que la idea básica que envuelve al concepto es la de ser un recurso individual y/o colectivo fundamentado y/o ubicado en una red de relaciones sociales ${ }^{4}$. Lozares, Verd y alters (2011), muestran que se trata de un concepto que combina, casi siempre sin estructurar, tres dimensiones conceptuales: (i) confianza, reciprocidad generalizada y solidaridad, accesibilidad, fiabilidad; (ii) relaciones y redes; (iii) normas sociales. Algunos autores añaden elementos como participación y compromiso, valores e identidad compartidos. Tomando lo precedente como base sustantiva, Lozares, Verd y alters (2011), apuntan la existencia de dos orientaciones fundamentales a la hora de concebir el capital social. Una que enfatiza sus contenidos atributivos, en la línea de Onyx y Bullen (2000), Portes (1998), Coleman (1988), Fukuyama (1995) o Putnam (1995; 2001). La otra orientación se centra más en los contenidos relacionales o reticulares, como por ejemplo Bourdieu (1986) aunque no formalmente en la idea de red, u otros más claramente centrados en la idea de red como Granovetter (1973), Burt (1992; 2000; 2001), Nahaliet y Goshal (1998), Woolcock y Narayan (2000), Borgatti, Jones y Everret (1998), Lin y Nan (1999) o Adler y Kwon (2002).

La evidente polisemia de los términos Cohesión social y Capital social conlleva algunas concepciones en que el campo semántico de sus definiciones es similar, por no decir paralelo: confianza mutua, reciprocidad y redes, extendiéndose a otros como reconocimiento, valores comunes, sentido de pertenencia, inclusión.

En nuestro caso, la Cohesión social se entiende como la expresión del Capital social interno de un grupo o colectivo que puede conllevar diferentes contenidos, por ejemplo confianza, reciprocidad, normas, identidad y valores, redes, etc. pero también otros de carácter cultural, político, civil, económico, de apoyo, de valores, etc. Como el Capital social, la Cohesión social contiene pues un contenido sustantivo y unas relaciones con mayor o menor intensidad; es decir, un componente formal en redes y/o relaciones.

\section{Bonding, Cohesión social y Capital social}

Así pues, la articulación del Capital social y de la Cohesión social proviene de identificar esta última, a partir de las relaciones Bonding, como un

${ }^{4}$ Para una revisión detallada del concepto véase Lozares, Verd, Lopez-Roldan Verd, Martí y Molina (2001).

EMPIRIA. Revista de Metodología de Ciencias Sociales. N. ${ }^{\circ}$ 26, julio-diciembre, 2013, pp. 35-62. ISSN: 1139-5737, DOI: 10.5944/empiria.26.7152 
recurso relacional y distribuido de Capital social en un colectivo de individuos, grupos, asociaciones o instituciones. Las relaciones son pues claves para identificar la Cohesión social y, por tanto, la forma de su distribución en cualquiera de sus posibles contenidos: cantidad, densidad, etc. Los Bonding se definen como relaciones hacia dentro y horizontales en tanto que forma de distribución del Capital social en un colectivo. Generan Cohesión social también por su componente formal según los contenidos que se consideren. Esta forma de distribución interna del Capital social, más o menos cohesiva, genera estructura social.

\subsection{Bridging/Linking ${ }^{5}$ como estructuras de la distribución del Capital social entre varios conjuntos o colectivos}

\section{Bridging/Linking y el Capital social}

Si las relaciones Bonding se refieren a un solo colectivo, las relaciones Bridging/Linking se refieren a las relaciones entre los agentes o entidades de dos o más colectivos, grupos, instituciones, asociaciones o poblaciones. Son relaciones hacia fuera, inter o entre colectivos tomando como referencia cada uno de los colectivos separadamente. Los colectivos están delimitados por fronteras o por características de los individuos. Estas relaciones van en sentido opuesto a las de clausura o de relaciones internas o Bonding. Las relaciones de intermediación entre colectivos o individuos diferentes facilitan los cambios de normas en el grupo propio, las alianzas exógenas, la competición o colaboración con diferentes, procuran nuevas fuentes de información e innovación y representan oportunidades para los intermediarios y/o los ocupantes de agujeros estructurales, tal y como lo examina Burt (1997). Además se asocian más a connotaciones instrumentales y competitivas que a expresivas y cohesivas (Johanson, 2001; Lin, 1999; 2001; Granovetter, 1973).

Los Bridging/Linking son una forma de expresión y estructuración reticular del Capital social que procura beneficios por posibilitar a los miembros de un grupo su acceso a grupos diferentes.

\section{Integración social y Capital Social}

Similar a lo que sucedía con la Cohesión social, la Integración social presenta también dificultades para su identificación dado el significado polivalente con que aparece en la literatura. La Integración social supone y se aplica a distintos colectivos sociales, previamente definidos, sea de modo intensivo por determinadas características o extensivamente por la delimitación de agentes, entidades o unidades. Es decir, la base de referencia es un conjunto de agentes con determinados roles, atributos, clases o posiciones pero también grupos, colectivos, aso-

${ }^{5}$ No se diferencia por ahora entre los conceptos de Bridging y Linking. Ambos son tomados como Bridging. Véase más adelante la nota 6.

EMPIRIA. Revista de Metodología de Ciencias Sociales. N. ${ }^{\circ}$ 26, julio-diciembre, 2013, pp. 35-62. ISSN: 1139-5737, DOI: 10.5944/empiria.26.7152 
ciaciones, instituciones, organizaciones, etc. La Integración social se ha relacionado con la idea de pertenencia a roles diferentes, conjunción de la diversidad, inclusión, etc. sin obviar conflictos y discrepancias entre roles y/o personas. Las diferencias pueden reportar también beneficios mutuos a partir de la a complementariedad o diversidad. La Integración social ha sido considerada por Gough y Olofsson (1999) como relaciones positivas desarrolladas y adquiridas sobre todo en la diversidad que procuran los lugares de trabajo, escuela, vecinos, etc. Sus contenidos, como en la Cohesión, pueden ser económicos, culturales, afectivos, etc. (Entzinger y Biezeveld, 2003).

\section{Bridging/Linking ${ }^{6}$, Integración social y Capital social}

La Integración social consiste, como la Cohesión, en la conjunción de una forma y de unos contenidos de diferentes campos sociales. Además, como en la Cohesión, se han de identificar previamente las unidades de referencia que son sujeto de las relaciones y los colectivos entre los que se define la Integración social. La Integración social se puede referir (i) a las relaciones de un individuo con el colectivo de referencia elegido pe. de un profesor en el departamento de su universidad; (ii) de una asociación con el colectivo de asociaciones; (iii) de la clase media con el conjunto de las clases sociales de una población o sociedad; (iv) de un departamento en la universidad concebida como un conjunto de departamentos. Se trata pues de relaciones entre, inter o hacia fuera. Vranken (2001) habla de las relaciones Bridging/Linking como interacciones o relaciones que se establecen entre colectivos o grupos a partir de sus relaciones. Las relaciones de Integración social son también una forma de distribución de Capital social de un individuo en un conjunto de individuos con respecto a un colectivo o de un grupo en un conjunto de grupos o colectivos. La Integración social puede ser gradual o de diferente intensidad y los contenidos también diversos: cognitivo de representaciones mutuas y/o de la inteligencia emocional, en tanto que terrenos de la confianza; de objetivos e instrumentales en las comunidades de prácticas; económicos; de la solidaridad a partir del apoyo mutuo, etc. En definitiva, los Bridging/Linking son adecuados indicadores de la Integración social, en tanto que son relaciones hacia fuera o externas o entre colectivos. Como tal es una forma de distribución del Capital social desde una perspectiva relacional.

${ }^{6}$ En determinados autores con una visión más reticular del Capital social y de la Integración social se distinguen dos tipos de relaciones hacia fuera, las denominadas hasta ahora como Bridging/Linking. De este modo se habla de dos tipos de Integración social: la Vinculación social o de Bridging y la Integración social, propiamente dicha o de Linking (Woolcock, 2000, 2001; Woolcock y Narayan, 2000; Szreter y Woolcock, 2004; Cheong, Edwards, Goulbourne y Solomos, 2007). En este artículo expresamos estas dos versiones de una manera más amplia con el único término de Bridging/Linking.

EMPIRIA. Revista de Metodología de Ciencias Sociales. N. ${ }^{\circ}$ 26, julio-diciembre, 2013, pp. 35-62. ISSN: 1139-5737, DOI: 10.5944/empiria.26.7152 


\subsection{Los Bonding y Bridging/Linking como formas de operativizar la Cohesión y la Integración en el marco del Capital social}

Como se ha mostrado en los puntos precedentes, la Cohesión e Integración se pueden aplicar como conceptos a uno o varios colectivos previamente definidos o identificados en el proceso mismo del análisis. Planteamos a continuación las posibilidades de operativización de dichos conceptos en función de que se considere un solo colectivo o se consideren colectivos o grupos diferenciados.

Si consideramos un solo colectivo de partida como universo poblacional, sin tener en cuenta grupos específicos internos, las relaciones de sus unidades, dentro o intra, del colectivo definen su Bonding. La Cohesión se caracteriza entonces, además de por otros indicadores, por la densidad de relaciones dentro del colectivo. En este caso no tiene sentido considerar la Integración pues se tiene sólo en cuenta un colectivo. Sólo es posible comparar los grados de Cohesión entre diferentes colectivos por la intensidad de sus Bonding respectivos, y desde la perspectiva tomada, como forma de distribución de Capital social.

$\mathrm{Si}$ se considera un colectivo configurado por grupos diferenciados existen dos posibilidades: la primera, calcular los Bonding o Cohesión de cada grupo interno y compararlas entre sí; la segunda, calcular los Bridging/Linking entre los grupos y con ello la Integración de todo el colectivo. Estas relaciones las identificamos como Linking/Bridging en tanto que indicadores de la Integración de unos grupos con otros en la totalidad de un colectivo.

\section{MODALIDADES DE COHESIÓN-INTEGRACIÓN: RELACIONAL Y RELACIONAL-ATRIBUTIVA}

Como se anuncia en la introducción, se presentan dos modalidades de la Cohesión-Integración. No se trata de opciones alternativas o concepciones opuestas sino más bien complementarias, en el sentido de que en la primera modalidad sólo se tendrá en consideración los criterios relacionales o reticulares y en la segunda se superpondrán a dicha relación las características o atributos sociales de los agentes.

El concepto de Cohesión-Integración, como se viene afirmando, consiste en una distribución del Capital social bajo las formas reticulares de Bonding- Bridging/Linking. Los agentes o entidades de los colectivos provienen o son elegidos por criterios nominales, atributivos o territoriales.

Tanto la Cohesión como la Integración se miden por la densidad de las relaciones, intra para la Cohesión e inter para la Integración. No se miden necesariamente, en primera instancia, por lazos fuertes, en el sentido por ejemplo de contenidos de confianza para la Cohesión o instrumentales para la Integración. De esta manera, en la Cohesión o Integración la intensidad de las relaciones son trasversales para cualquier contenido lo que permite su medición, comparación y su extensión a cualquier ámbito social. 
La estructura de la Cohesión-Integración es pues relacional, esto es, exclusivamente reticular y su contenido conceptual correspondiente es diverso. La Cohesión relacional proviene de las relaciones entre agentes o entidades de un conjunto o colectivo previamente definido. La Integración relacional proviene de las relaciones entre agentes o entidades de conjuntos diferentes. Con todo, ambas pueden ser analizadas separadamente o conjugadas. La Cohesión-Integración relacional constituye una estructura relacional que clasifica los agentes del colectivo.

Así, pues, entre identificar primero los atributos de los agentes y luego sus relaciones, la Cohesión-Integración relacional parte de las relaciones como origen y desarrollo del proceso. Como se expresa en el esquema 1 que se presenta seguidamente, la Cohesión-Integración social se origina en la interacción o relación entre agentes de tipo Bonding-Bridging para situar a los agentes en una estructura relacional con sus estatus o posiciones diferenciales.

Esquema 1. Proceso en la perspectiva relacional-reticular o versión estricta

\begin{tabular}{|l|l|l|}
$\substack{\text { Cohesión-Integración } \\
\text { Bonding-Bridging/Linking }}$ & Colectivos & $\begin{array}{l}\text { Estructura relacional: } \\
\text { agentes en posiciones }\end{array}$ \\
reticulares
\end{tabular}

En la segunda versión, que denominamos relacional-atributiva, se parte de la Cohesión-Integración relacional precedente pero haciendo intervenir de manera complementaria las características de los agentes. Con ello, se da identidad atributiva a la estructura relacional en caso de que exista una homología entre la estructura reticular de los agentes y la de sus atributos. La información adicional proviene de los datos atributivos de cariz socio-demográficos u otros.

Según el procedimiento seguido se presentan diferentes posibilidades de análisis. Si se trata de redes socio-métricas, las diferentes posiciones de los agentes, sea en grupos cohesivos sea por sus relaciones integrativas, se identifican con sus características atributivas. Si se trata de redes ego-centradas, se parte de las relaciones entre los Alteri de cada Ego (que provienen de las interacciones entre Alteri), lo que genera propiedades relacionales en cada red de Alteri según su Cohesión-Integración. Dichas propiedades son atribuibles a cada Ego de tal manera que generan clasificaciones de los Egos según el grado de Cohesión-Integración de la red de sus Alteri. Por asociación o correlación es posible analiz grado de homología existente entre dicha clasificación relacional de los Egos con sus atributos o características sociales. El Esquema 2 explicita el proceso para las redes Ego-centradas pero es válido también para las redes socio-métricas ${ }^{7}$.

${ }^{7}$ El esquema es válido para las redes socio-métricas. En este caso se entiende que en vez de referirse a los Alteri de cada Ego para establecer y analizar las relaciones de la red se refiere a los Egos y sus relaciones en la red socio-métrica.

EMPIRIA. Revista de Metodología de Ciencias Sociales. N. ${ }^{\circ}$ 26, julio-diciembre, 2013, pp. 35-62. ISSN: 1139-5737, DOI: 10.5944/empiria.26.7152 
Esquema 2. Proceso de la Cohesión-Integración en versión relacional-atributiva

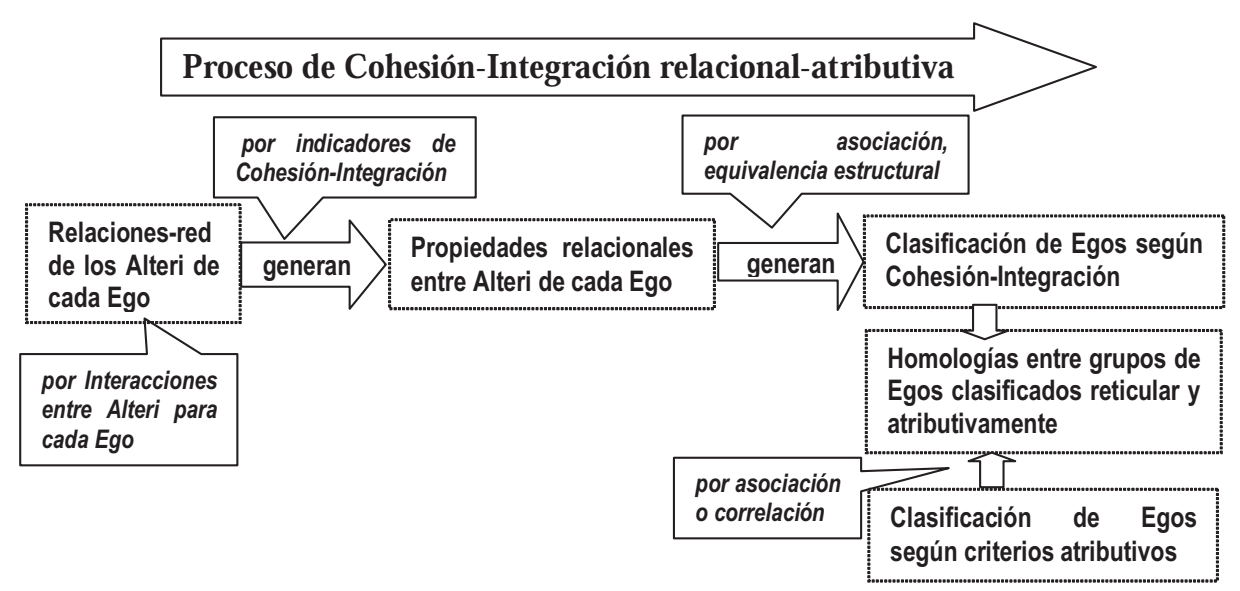

\section{LOS DATOS, EL TIPO DE ENCUESTA, LOS COLECTIVOS Y LOS SUPUESTOS}

Los datos empíricos analizados provienen de una encuesta de redes egocentradas llevada a cabo durante los últimos meses del año 2009 y los primeros del año 2010. Se realizó a partir de una muestra aleatoria, estratificada por cuotas -de los colectivos y ciudades indicados seguidamente-, bietápica (Lozares et al., 2011) y con presencia equitativa por género. La encuesta recoge información de 447 Egos, sobre sus datos socio-demográficos u otros de interés, sobre sus relaciones con otros individuos (llamados Alteri), características de dichos Alteri y las relaciones que mantienen entre sí los Alteri. La encuesta ego-centrada llevada a cabo, a diferencia de otros tipos de encuestas de esta naturaleza, contiene un gran número de preguntas de tipo atributivo, tanto para los Egos como para sus Alteri. Ello permite ampliar el campo de temas que son objeto de estudio en la Cohesión e Integración entre colectivos. Además, también se extiende el contenido relacional entre Egos y Alteri con otros contenidos que se superponen al habitual de conocimiento y trato ${ }^{8}$.

Los colectivos que son objeto de investigación tienen que ver con tres tipos de población de Cataluña que se corresponden con dos oleadas migratorias y con la población autóctona, esto es: (i) la residente en Cataluña de padres nacidos en Cataluña; (ii) la proveniente de otros territorios de España en los años 1950 y 60

${ }^{8}$ Si no se puede hablar de una extensión representativa a toda Cataluña, sí que es posible que lo sea de cada colectivo, dado el carácter elevadamente homogéneo en muchas de sus características sociales de los miembros de cada colectivo; esto es, que sirvan de buena base para un estudio comparativo.

EMPIRIA. Revista de Metodología de Ciencias Sociales. N. ${ }^{\circ}$ 26, julio-diciembre, 2013, pp. 35-62. ISSN: 1139-5737, DOI: 10.5944/empiria.26.7152 
además, genéricamente, de la generación de sus hijos; (iii) la inmigración reciente de las que sólo se han elegido los colectivos de origen marroquí y ecuatoriano. Estos colectivos, que son la base de la estratificación muestral, han sido elegidos por dos razones. La primera, porque representan tres orígenes y momentos de incorporación a Cataluña y, en gran medida, como consecuencia, significan también tres modos de inserción en ámbitos sociales. La segunda, porque tomando diferentes colectivos permite no sólo estudiar la Cohesión de cada colectivo sino también su Integración mutua. En el caso de estos colectivos tanto la Cohesión como la Integración incumben a todos los colectivos.

De los tres colectivos indicados, dos de ellos, los compuestos por catalanes, están doblados por un criterio de edad, de 25 a 55 y más de 55; a la nueva inmigración no se aplica tal criterio pero se eligen dos colectivos muy diferenciados, el de los nacidos en Ecuador y los nacidos en Marruecos. Ello lleva a los seis colectivos definitivos siguientes:

- Catalanes nacidos en Cataluña, de padres catalanes, mayores de 55 años, desde ahora, $N C+55 p N C$

- Catalanes nacidos en Cataluña, de padres catalanes, entre 25 y 55 años, desde ahora, $N C$ 25-55pNC

- Catalanes nacidos en Cataluña, de padres nacidos en el resto de España, entre 25 y 55 años, desde ahora, $N C$ 25-55pNE

- Catalanes nacidos en el resto de España mayores de 55 años, desde ahora, $N E+55$

- Origen en Ecuador menores del 55

- Origen en Marruecos menores de 55

La elección de estos colectivos es importante como criterio de estratificación para la muestra. Estudios de estructura social en Cataluña evidencian que los orígenes, inmigración y edad se corresponden con factores importantes discriminantes en Cataluña y, concretamente, con las categorías socio-profesionales (López-Roldán y Lozares, 2008a; López-Roldán y Lozares, 2008b). Además del origen y edad, se han elegido como criterio de estratificación de la muestra tres ciudades que por su desarrollo metropolitano, industrial, urbanístico, etc. en las últimas décadas son cotejables con otras muchas ciudades de Cataluña con similares características: se trata de Barcelona, Sant Feliu y Balaguer.

No obstante la gran variedad de análisis que se pueden llevar a cabo con las encuestas ego-centradas y en particular con la presente, en este artículo el objetivo consiste en un análisis comparado de la Cohesión-Integración de y entre los colectivos y, concretamente, los descritos. Si bien el objetivo específico de este artículo es sobre todo metodológico, sin embargo se extraen consecuencias con respecto a la Cohesión-Integración en colectivos determinados. En este sentido, el supuesto genérico es que los grupos cohesivos generados tienden a reproducir los colectivos atributivos por criterios de edad o de origen y que la integración de colectivos de origen diferente se hace por poseer edades en un mismo intervalo o viceversa.

EMPIRIA. Revista de Metodología de Ciencias Sociales. N. ${ }^{\circ}$ 26, julio-diciembre, 2013, pp. 35-62. ISSN: 1139-5737, DOI: 10.5944/empiria.26.7152 
Por otro lado, el tipo de diseño y contenidos de la encuesta procura la suficiente información como para extender el análisis a numerosos otros campos sociales y colectivos como la categoría socio-profesional, género, lengua, etc. o al cruce de dichos atributos, como también a otros contenidos relacionales, por ejemplo proximidad de amistad o el nivel de confianza, etc. la pertenencia a las mismas asociaciones, empresa, etc., soportes o ayudadas específicas, etc.

\section{LA APLICACIÓN Y EL ANÁLISIS}

En este apartado se examinan y analizan los diferentes modos de CohesiónIntegración en cada colectivo y entre sí por medio de redes socio-métricas, ego-centradas y su combinación. En esta aplicación se unifican los objetivos sustantivos: el primero por el estudio de los diferentes modos de cohesión e integración en los distintos colectivos; el segundo, de naturaleza metodológica por un diseño combinado de redes socio-métricas y ego-centradas; y, el tercero, por los métodos de análisis y uso de conceptos-indicadores de las redes sociales aplicados a los colectivos.

En la primera parte 4.1 se analizan la Cohesión-Integración relacional (apartado 4.1.1) y la relacional-atributiva en las redes socio-métricas (apartado 4.1.2). En la segunda parte 4.2 se analiza también ambas formas de CohesiónIntegración pero en redes ego-centradas.

\subsection{La concepción relacional y la relacional-atributiva de la Cohesión- Integración en redes socio-métricas.}

\subsubsection{La Cohesión-Integración relacional en redes socio-métricas.}

Se parte de un colectivo de agentes que mantienen entre sí sus relaciones mutuas, dirigidas o no. Este conjunto está delimitado sea de modo nominal o realista. Los agentes informan, o son observados, sobre las relaciones que se establecen dentro del colectivo y ello para todos sus agentes. La red de relaciones establece una estructura y, por tanto, posiciones, diferenciadas o no, de los agentes en dicha red. Dentro de la versión estrictamente reticular no se consideran primariamente los atributos. Si se parte de una red socio-métrica como la presente (Gráfico 1) hay dos posibilidades: (a) considerar la totalidad de la red como un solo colectivo. Si no interesa calcular su estructuración interna sino sólo su cohesión global, es apropiado calcular su densidad ${ }^{9}$ global como indicador de su Bonding; en este caso no tiene sentido calcular la comparación de la Cohesión entre colectivos porque son considerados como un todo, ni tampoco tiene sentido calcular la Integración entre ellos. (b) Considerar diversos colectivos en el inte-

${ }^{9}$ La densidad de una red se define como la proporción de relaciones existentes entre sus nodos con respecto a todas las posibles.

EMPIRIA. Revista de Metodología de Ciencias Sociales. N. ${ }^{\circ}$ 26, julio-diciembre, 2013, pp. 35-62. ISSN: 1139-5737, DOI: 10.5944/empiria.26.7152 
rior de la red socio-métrica, en el caso como dos grupos. En este caso, es posible calcular la Cohesión de los diferentes grupos así como la Integración entre ellos. Los grupos de agentes en el interior del colectivo se establecen por criterios reticulares. Para identificar los grupos reticulares se pueden usar diversos criterios, desde las Cliques hasta algoritmos como los de Girvan-Newman, Faction u otros con resultados idénticos. En nuestro caso se usa el de Faction ${ }^{10}$, que da un primer colectivo con 9 personas y un segundo con 21 (Tabla 1; Gráfico 1). La Tabla 1 muestra, para un Ego de origen marroquí, las densidades o Bonding de cada grupo: 1 y 0.97 ; los valores de la diagonal invertida son nulos, esto es, la Integración expresada en Bridging/Linking.

Tabla 1. Densidad de Grupos

\begin{tabular}{rcc}
\hline Grupos & Grupo 1 & Grupo 2 \\
\hline Grupo 1 & 1.00 & 0.00 \\
Grupo 2 & 0.00 & 0.97 \\
\hline
\end{tabular}

\section{Gráfico 1 ${ }^{11}$. Grupos generados}

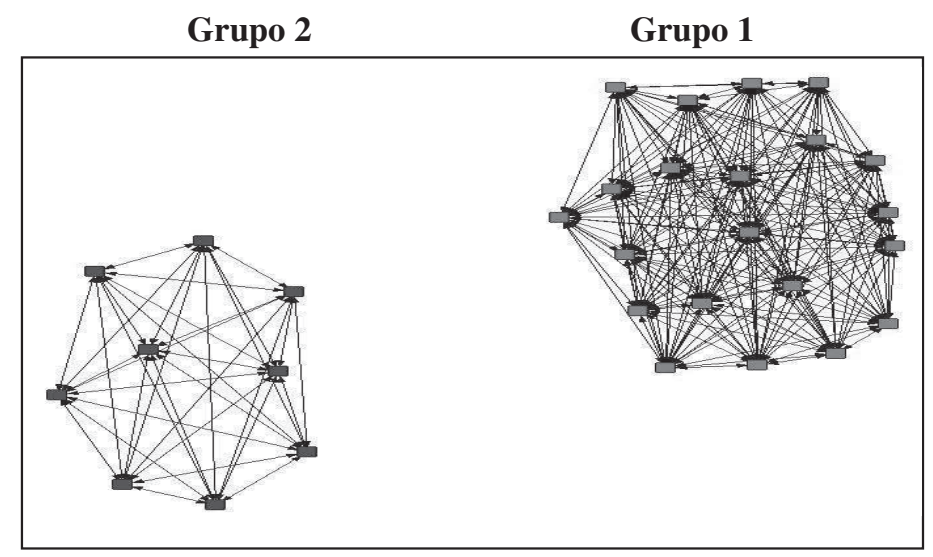

Atendiendo al criterio elegido, la identidad reticular de los grupos viene dada por indicadores que miden la Cohesión e Integración. La Cohesión relacional de un grupo es 1, que expresa la máxima densidad posible; y la del otro grupo es 0.97, que significa una muy elevada densidad. La Integración mutua en este caso es nula pues no hay relaciones inter o entre los grupos.

${ }^{10}$ El criterio Faction establece los colectivos o grupos de tal manera que sus relaciones internas o intra en cada grupo sean más densas que las relaciones inter grupo.

${ }^{11}$ Ha sido elegido como ejemplificación el caso de una red socio-métrica de los Alteri de un Ego de origen marroquí.

EMPIRIA. Revista de Metodología de Ciencias Sociales. N. ${ }^{\circ}$ 26, julio-diciembre, 2013, pp. 35-62. ISSN: 1139-5737, DOI: 10.5944/empiria.26.7152 


\subsubsection{Las Cohesión-Integración relacional-atributiva en las redes socio-métricas}

Como se viene anunciando, el análisis de la Cohesión-Integración relacionalatributiva parte de los cálculos precedentes tratando de identificar la naturaleza atributiva de cada uno de los colectivos generados por la vía reticular. Se trata de superponerles un «nombre» y sus características reticulares; es decir, de conjugar lo relacional con lo atributivo examinado la correspondencia entre estructura relacional y atributiva.

Solamente se hacen los cálculos para identificar los grupos de alteri por los colectivos de origen, si bien se podrían identificar a partir de una gran variedad de variables (género, profesión, nivel de estudios, etc.) (Gráfico 2; Tabla 2).

Tabla 2. La identidad de los Grupos

\begin{tabular}{lccc}
\hline \multicolumn{1}{c}{ Atributos } & Grupo 1 & Grupo 2 & Total \\
\hline NC+55pNC & 1 & 0 & 1 \\
Ecuador & 2 & 0 & 2 \\
Marruecos & 6 & 21 & 27 \\
Total & 9 & 21 & 30 \\
\hline
\end{tabular}

Grafico 2. Redes de los Grupos

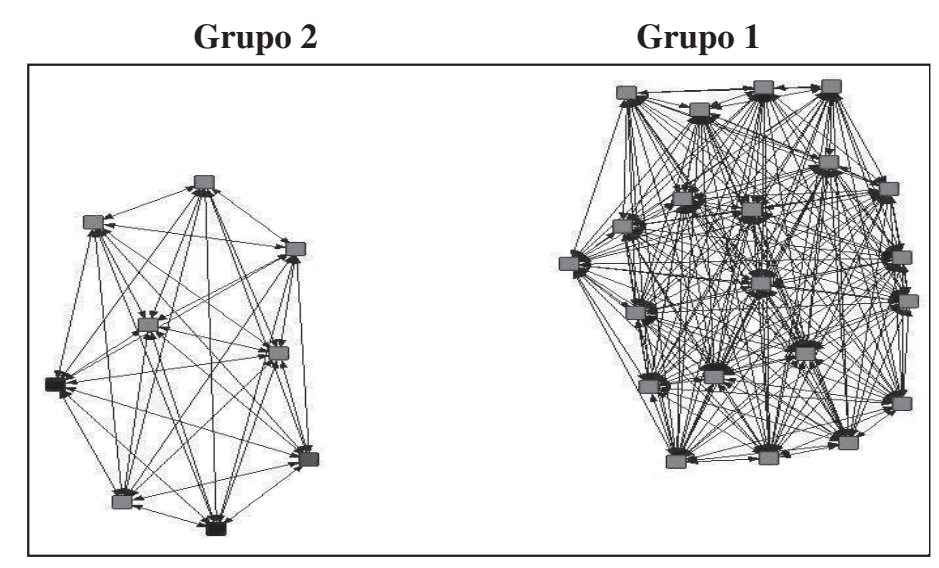

El primer grupo, menos denso, está formado por 9 personas con la distribución siguiente: una persona $\mathrm{NC}+55 \mathrm{pNC}$, dos Ecuatorianos y 6 Marroquíes; en el segundo grupo con 21 personas y mucho más denso todos son marroquíes. El primer grupo algo menos denso contiene además de marroquíes otras dos nacionalidades; puede ser, en ausencia de otras informaciones también disponibles pero no analizadas, que se trate de un colectivo vinculado a la vida laboral. El 
grupo más denso y numeroso parece más bien ligado a la vida familiar y amical con todos los miembros marroquíes.

\subsection{La concepción relacional y la relacional-atributiva de la Cohesión- Integración en redes ego-centradas}

Dentro de las redes ego-centradas, se presentan a continuación dos aplicaciones diferentes en el modo de vincular, en el sentido de la CohesiónIntegración, las características atributivas de los Egos con los grupos formados con indicadores reticulares de las redes de los Alteri de cada Ego. La diferencia entre ambas aplicaciones consiste en que en la primera se considera un conjunto de indicadores en las redes socio-métricas de cada Ego que dan cuenta de la estructuración interna de las redes, dentro de las posibilidades que van de la integración a la segregación (sub-apartado 4.2.1); en la segunda aplicación se sigue un solo criterio global reticular en la dirección de la Cohesión de las redes socio-métricas de los Egos para asociarlos a los colectivos (sub-apartado 4.2.2).

\subsubsection{Una primera aplicación y diseño de la concepción relacional y relacional-atributiva de la Cohesión-Integración en redes ego-centradas por el análisis de la red de los Alteri para cada Ego.}

Como se ha anunciado, en este diseño se generan grupos por características estrictamente relacionales que se asocian por contingencias con los colectivos que provienen de las variables atributivas. El procedimiento general consiste: primero, en su versión relacional estricta (subapartado a), en analizar internamente las redes socio-métricas de los Alteri para cada Ego por medio de indicadores del análisis de redes que expresen las formas y grado de articulación de los grupos dentro de la red, en el sentido de la integración a la segregación; y, segundo, en su versión relacional-atributiva (subapartado b), en asociar dichos grupos a los colectivos de Egos basados en criterios atributivos a fin de identificar los grupos también por estas dichas características atributivas.

\section{(a) La perspectiva relacional}

Se parte de las matrices socio-métricas de los Egos, es decir, de las relaciones entre sus Alteri para cada uno y todos los Egos. El contenido de dicha relación es de conocimiento y trato mutuo. En dichas redes se calcula la CohesiónIntegración relacional por criterios relacionales.

Se trata de analizar las redes socio-métricas de cada Ego tomando en cuenta sus estructuras internas para identificar los grupos formados relacionalmente en cada matriz socio-métrica. El cálculo se extiende a todas las matrices 
socio-métricas de los Egos para agruparlos según la similitud de la estructura de grupos formados en las redes socio-métricas de los Egos. En este diseño se aplican indicadores globales que dan cuenta de la estructuración interna de las redes: $\mathrm{n}^{\mathrm{o}}$ de nodos aislados, $\mathrm{n}^{\mathrm{o}}$ de componentes, $\mathrm{n}^{\mathrm{o}}$ de diadas $\mathrm{y} \mathrm{n}^{\mathrm{o}}$ de cliques $^{12}$. Los tres primeros, según sus valores, son indicativos de una red poco estructurada internamente o segmentada al límite o, del otro, de una red más entretejida de grupos articulados e internamente cohesivos. La tendencia, en la primera opción, va más en el sentido de una menor articulación y mayor segregación de la red, mientras en la segunda la tendencia es de una mayor Cohesión global o de equilibrio Cohesión-Integración. Como análisis previo se examinan dichos indicadores para todas las matrices dimensionando dicho cálculo en una combinación factorial; los factores condensan así la información, de modo que se obtiene la estructura interna de las matrices socio-métricas de todos los Egos (Tabla 3).

Tabla 3. Análisis factorial varianza, representación, factores y validación

\begin{tabular}{cccccccccc}
\hline \multicolumn{4}{c}{ Autovalores iniciales } & \multicolumn{3}{c}{ Suma saturación abstracción } & \multicolumn{3}{c}{ Suma saturación rotados } \\
\hline \multirow{2}{*}{ Factor } & Total & $\begin{array}{c}\% \\
\text { Varianza }\end{array}$ & $\begin{array}{c}\% \\
\text { suma }\end{array}$ & Total & $\begin{array}{c}\% \\
\text { Varianza }\end{array}$ & $\begin{array}{c}\% \\
\text { suma }\end{array}$ & Total & $\begin{array}{c}\% \\
\text { Varianza }\end{array}$ & $\begin{array}{c}\% \\
\text { suma }\end{array}$ \\
\hline 1 & 1,501 & 37,520 & 37,520 & 1,501 & 37,520 & 37,520 & 1,412 & 35,302 & 35,302 \\
2 & 0,927 & 23,171 & 60,692 & 0,927 & 23,171 & 60,692 & 1,016 & 25,390 & 60,692 \\
3 & 0,898 & 22,443 & 83,134 & & & & & & \\
4 & 0,675 & 16,866 & 100,000 & & & & & & \\
\hline
\end{tabular}

\begin{tabular}{lcc}
\cline { 2 - 3 } & \multicolumn{2}{c}{ Factores } \\
\cline { 2 - 3 } & 1 & 2 \\
\hline N Nodos aislados &, 750 &,- 131 \\
N Componentes &, 689 &,- 187 \\
N Diadas &, 609 &, 136 \\
N_Cliques & &, 972 \\
\hline
\end{tabular}

${ }^{12}$ Estos conceptos son propios de la teoría y análisis de redes sociales: «nodos aislados» son Alteri sin relación con otros Alteri; «componentes» son grados aislados de Alteri comunicados entre sí pero separados del resto de la red; «diadas» son pares de relaciones entre dos Alteri; «cliques», de una forma general, pueden definirse como subconjuntos de agentes de una red que están más cercanos, esto es, más conectados o comunicados mutuamente, que lo que lo están con el resto de integrantes de una red. De forma más concreta, son conjuntos de Alteri comunicados entre sí a una distancia determinada (la distancia 1 es la más exigente y daría las cliques más densas), pudiendo las cliques estar comunicadas con el resto de los Alteri de la red. 
Los dos ejes marcan una diferencia clara e independiente en los comportamientos de los indicadores de estructuración en las redes socio-métricas. El primer eje representa, del lado positivo, la tendencia a la dispersión, segmentación y segregación de la red; y de su lado negativo, su ausencia. El segundo eje representa, del lado positivo, una configuración o completamente densa, para el caso máximo, pasando por un equilibrio entre Cohesión e Integración; y del lado negativo, la articulación mínima de grupos internos ( $\mathrm{n}^{\circ}$ de cliques). Como se afirma dichos ejes funcionan de manera independiente.

Establecidos los ejes, el proceso consiguiente consiste en agrupar Egos en función de los factores hallados en el comportamiento reticular de las matrices reticulares de sus Alteri. El objetivo es encontrar grupos de Egos que internamente sean lo más similares en dichas características factoriales-reticulares y lo más diferentes entre sí. Así, el grupo y la identidad que emerja para cada grupo vendrán dados sólo por criterios factoriales-reticulares. En vistas a una elección más adecuada se hacen análisis de Cluster entre 6 y 2 conglomerados. En la Tabla 4 los números que siguen al nombre de los grupos indican el $n^{\circ}$ de Egos, y por tanto de matrices, presentes en cada grupo.

Tabla 4. Generación de Grupos y sus valores factoriales; tablas y gráfico

\begin{tabular}{ccc}
\hline 6 Grupos & factor 1 & \multicolumn{1}{c}{ factor 2} \\
\hline G1 280 &,- 5449701 &,- 1123376 \\
G2 109 &, 5063903 &,- 2096798 \\
G3 36 & 2,7431338 &,- 2272229 \\
G4 9 &,- 2410736 & 1,8295875 \\
G5 6 &, 0313521 & 5,7251188 \\
G6 1 &, 6238148 & 11,6726639 \\
\hline 4 Grupos & factor 1 & factor 2 \\
\hline G1 289 &,- 5355062 &,- 0518625 \\
G2 109 &, 5063903 &,- 2096798 \\
G3 36 & 2,7431338 &,- 2272229 \\
G4 4 &, 1159897 & 6,5747681 \\
\hline 3 Grupos & factor 1 & factor 2 \\
\hline G1 398 &,- 2501627 &,- 0950838 \\
G2 36 & 2,7431338 &,- 2272229 \\
G3 7 &, 1159897 & 6,5747681 \\
\hline
\end{tabular}




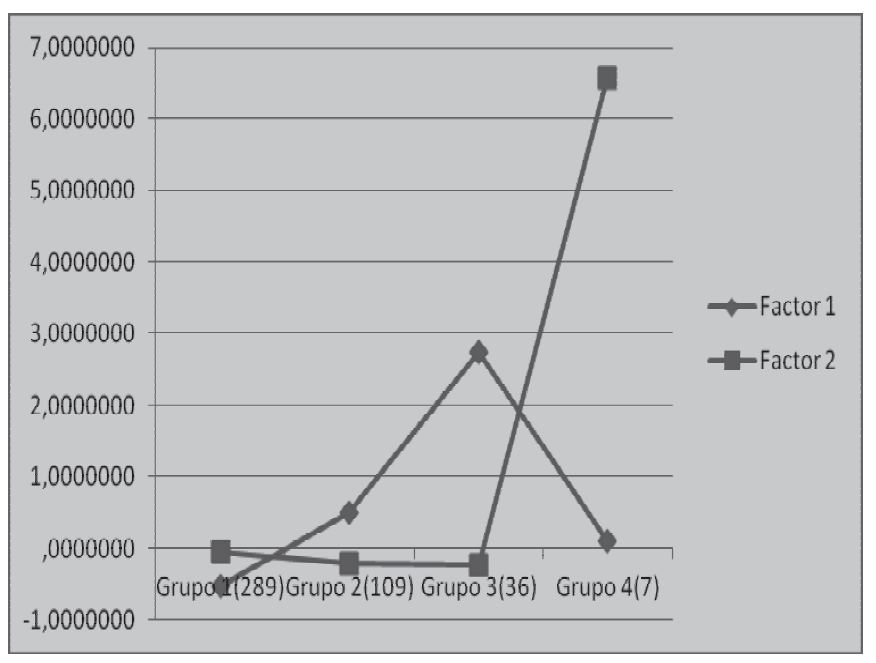

Aunque se ha llevado a cabo una reducción sucesiva del número de grupos, ésta no conlleva una distribución más equitativa del número de Egos (o matrices) en los grupos. Casi todos los Egos se acumulan en los dos primeros grupos, si bien los ejes son claramente discriminantes. El análisis se hace con 4 grupos que, aunque no equilibrados numéricamente, logran una mejor redistribución de los grupos en cuadrantes diferentes con respecto a los ejes. Veamos su identidad como estructura reticular.

Dentro de esta solución, el Grupo 1 mantiene un valor negativo en el primer eje y es prácticamente central en el segundo; esto es, en el primero, la ausencia de dispersión, segmentación y segregación de sus redes; en el segundo eje, su posición está más centrada con relación a la posesión de cliques: parece estar en una situación de equilibrio entre Cohesión e Integración. Dentro de esta tendencia y tratándose de 298 redes o Egos, este grupo ocupa una relativa posición central o media en el equilibrio entre dispersión e integración y/o cohesión.

El Grupo 2, con 109 redes o Egos, tiene un valor positivo en el primer eje, esto es, una tendencia a la dispersión, segmentación y segregación de sus redes; en el segundo, su valor aunque menor es negativo, es decir, tiene una tendencia a la ausencia de cliques o a una poca articulación de sus grupos internos.

El Grupo 3, con 36 Egos o matrices, acentúa enormemente su dispersión, segmentación y segregación con relación al segundo grupo y es similar en su tendencia a la ausencia de cliques, o poca articulación de sus grupos internos.

El Grupo 4 es un apéndice casi testimonial de sólo 4 Egos y se caracteriza por un altísimo valor en el segundo eje, esto es, por su articulación y compacidad por las cliques.

La conclusión evidente es que, aunque se hayan generado estos cuatro grupos diferenciados e identificado su identidad por sus valores en los ejes, el número de 
Egos en cada grupo no está distribuido equitativamente. Ello conduce a centrar más la interpretación en los dos primeros grupos, considerando los Grupos 3 y 4 como apéndices con pocos Egos y con sus características pero que, dado sus valores extremos en los ejes, contribuyen a centrar más los Grupos 1 y 2.

\section{(b) La versión relacional-atributiva.}

Se trata de examinar las equivalencias u homologías entre los grupos precedentes generados por criterios relacionales con grupos de Egos que corresponde a los colectivos Origen x Edad. Ello se lleva a cabo por medio de un análisis de asociación de contingencias entre colectivos y grupos tomando el Residuo corregido como indicador de asociación para hallar las equivalencias. De todas maneras, y dado los grupos que han emergido, es ya previsible que no haya una correspondencia neta entre los 6 colectivos y los 4 clusters o grupos. Considerando la identidad de los grupos a partir de los dos factores, indicativos de Cohesión-Integración o su ausencia, lo coherente es que esta identificación de los grupos por los colectivos lleve a la agrupación de éstos en tres o cuatro pautas de comportamiento.

Tabla 5. Asociación Grupos y Colectivos: identidad de los Grupos por Colectivos

\begin{tabular}{crrrrrrrr}
\hline \multicolumn{10}{c}{ Colectivos. Recuento y Residuos corregidos } \\
\hline $\begin{array}{c}\text { Grupos } \\
\text { reticulares corregidos RC }\end{array}$ & $\begin{array}{r}\text { Residuos } \\
\text { pNC }\end{array}$ & NC25- & 55NC & NC25- & 55pNE & NE+55 & Ecuador Marruecos & \multirow{2}{*}{ Total } \\
\hline 1 & Recuento & 55 & 44 & 46 & 50 & 43 & 50 & 288 \\
& RC & 1,9 & $-1,5$ &,- 7 & 1,3 & $-1,3$ &, 2 & \\
2 & Recuento & 11 & 24 & 22 & 13 & 22 & 17 & 109 \\
& RC & $-2,1$ & 1,5 & 1,1 & $-1,2$ & 1,2 &,- 5 & \\
3 & Recuento & 7 & 7 & 4 & 4 & 8 & 6 & 36 \\
& RC &, 5 &, 4 & $-1,0$ &,- 8 &, 9 &,- 1 & \\
4 & Recuento & 0 & 1 & 2 & 2 & 0 & 2 & 7 \\
& RC & $-1,2$ & $-2,0$ &, 8 &, 9 & $.1,2$ &, 8 & \\
Total & Recuento & 73 & 76 & 74 & 69 & 73 & 75 & 440 \\
\hline
\end{tabular}

El nivel de asociación de un supuesto acoplamiento entre los grupos de los Egos que provienen de las relaciones de sus Alteri con los colectivos de egos son más bien débiles: cuando algún dato es positivo no llega a ser significativo (más 1.96 y menos de -1.96 del residuo corregido). Veamos unas conclusiones orientativas (Tabla 5).

En el Grupo 4, con 7 Egos, no hay ninguna asociación significativa con colectivos; sólo existe una asociación débil no significativa con marroquíes, 
NE+55 y NC25-55pNE. El Grupo 3, tampoco manifiesta una asociación significativa con ningún colectivo (corresponde a 36 Egos); la asociación es sólo testimonial con ecuatorianos, NC+55pNC y NC25-55pNC. Ambos grupos son los de menos población.

El Grupo 1 está mayormente asociado con NC+55pNC y NE+55 y, por tanto, con personas mayores, sean de origen catalán o español fuera de Cataluña. Esto es, el criterio de edad es básico en la asignación del grupo a los colectivos. Este Grupo 1 está caracterizado desde el punto de vista reticular, primero, por la ausencia de dispersión, segmentación y segregación de sus redes; y, segundo, por una posición más centrada con relación a la presencia de cliques y una situación de equilibrio entre Cohesión e Integración. El Grupo 2 está más asociado y, por tanto, más mayoritariamente compuesto de NC25$55 \mathrm{pNC}$, NC25-55pNE y ecuatorianos; así, de nuevo la edad es lo que agrupa a dos colectivos de diferentes procedencias, aunque ambos son catalanes de nacimiento. Las características del Grupo 2 consisten, primero, en un valor positivo en su tendencia a la dispersión, segmentación y segregación de sus redes; y, segundo, a una menor presencia de cliques, esto es, a una poca articulación de sus grupos internos. La agrupación de estos colectivos (uno como $\mathrm{NC}+55 \mathrm{pNC}$ y $\mathrm{NE}+55$ y el otro como NC25-55pNC y NC25-55pNE) por criterios de edad para el análisis de asociación con los grupos reticulares hubiera procurado más intensidad a los índices de residuos corregidos y seguramente significatividad.

\subsubsection{Una segunda aplicación y diseño de la concepción relacional y relacional-atributiva de la Cohesión-Integración en redes Ego-centradas por el análisis de densidad en las redes de los Alteri para cada Ego.}

En este segundo diseño se generan grupos también por características estrictamente relacionales, en concreto, por la densidad, dado que es un indicador de Cohesión global de la red en menoscabo de las segregaciones o nodos aislados. Una vez generados e identificados dichos grupos por su densidad relativa se analiza su grado de asociación con los colectivos atributivos de los Egos por análisis de contingencias.

\section{(a) La perspectiva relacional}

El indicador usado es la densidad global para el conjunto de las redes sociométricas de todos los Egos. Con la variable densidad se lleva a cabo un análisis de conglomerados, maximizando la homogeneidad interna de cada grupo y tomando seis grupos para su mejor comparación con el número de los colectivos. Calculando la media de densidades para cada grupo se obtienen los resultados de la Tabla 6. 
Tabla 6. Densidad de Grupos

\begin{tabular}{ccc}
\hline Grupos & $\begin{array}{c}\text { Densidad } \\
\text { Media }\end{array}$ & $\begin{array}{c}\mathrm{N}^{\circ} \\
\text { Egos }\end{array}$ \\
\hline G1 & 26,04 & 73 \\
G2 & 49,78 & 91 \\
G3 & 36,56 & 137 \\
G4 & 17,05 & 51 \\
G5 & 66,34 & 70 \\
G6 & 92,41 & 18 \\
Total & 42,41 & 440 \\
\hline
\end{tabular}

Los grupos que surgen no tienen la misma distribución del $n^{\circ}$ de Egos, pero tampoco se da una acumulación excesivamente marcada en determinados grupos. Por orden decreciente en densidad, los grupos son los siguientes: G6, G5, G2, G3, G1 y G4. Desde el punto de vista de la Cohesión-Integración, los grupos de más densidad son aquellos en que las relaciones entre Alteri son más cohesivas, en cambio; los grupos menos densos son aquellos en que las relaciones entre Alteri son menos compactas, y por lo tanto sus contactos mutuos dependen más del Ego.

\section{(b) La versión relacional-atributiva}

El paralelismo asociativo es genérico y puede no estar necesariamente ajustado de forma idéntica, en completo isomorfismo. La razón está en que los grupos generados relacionalmente provienen de la agrupación por optimización de densidades, mientras que los colectivos están fijados previamente. Veamos dicha conjugación entre grupos y colectivos por un análisis de contingencia con el índice de Residuos corregido como indicador (Tabla 7).

Tabla 7. Residuos corregidos de asociación de Grupos con Colectivos

\begin{tabular}{lrrrrrr}
\hline \multirow{2}{*}{ Grupo y $\mathrm{n}^{\circ}$} & NC+ & NC25- & NC25- & NE+55 & \multirow{2}{*}{ Ecuador } & \multirow{2}{*}{ Marruecos } \\
\hline G1 (73) &,- 4 & 3,2 & $-1,5$ & $-2,3$ & 1,3 &,- 5 \\
G2 (91) &, 3 & $-1,5$ &, 8 &, 9 &, 6 & $-1,1$ \\
G3 (137) & $-1,6$ &,- 7 & 1,1 & 1,0 &,- 5 &, 7 \\
G4 (51) &, 6 &, 9 &,- 6 &,- 8 &,- 2 &, 1 \\
G5 (70) & 1,9 & $-1,1$ &, 4 &, 0 & $-1,6$ &, 4 \\
G6 (18) &,- 6 &,- 7 & $-1,3$ & 1,4 &, 7 &, 6 \\
\hline
\end{tabular}


La mayor parte de los valores de los residuos corregidos no son significativos, lo que va en el sentido de una escasa convergencia entre grupos generados relacionalmente y los colectivos preestablecios. Por ello, sólo concluimos sobre tendencias. Veamos las pautas de asociación según el orden decreciente en densidad para los grupos: G6, G5, G2, G3, G1 y G4.

El Grupo 6 se corresponde más con el colectivo NE+55 y el Grupo 5 con el colectivo NC+55pNC. Dichos colectivos, que son de más de 55 años con independencia de su origen de nacimiento, son también los más densos y, por tanto, los más cohesivos.

El Grupo 3 se corresponde con el colectivo NC25-55pNE y, algo más débilmente, con el NE+55. El Grupo 2 se corresponde débilmente con los colectivos NC25-55pNE, NE+55 y Ecuador. Son grupos menos cohesivos que los precedentes dado el valor de su densidad. Estos grupos aglutinan a colectivos de catalanes, sean nacidos en Cataluña de padres no nacidos Cataluña o catalanes nacidos en el resto de España. Son grupos menos densos que los precedentes (G6 y G5) y, por tanto, menos cohesivos.

El Grupo 1 se corresponde con el colectivo NC25-55pNC y, más débilmente, con el de ecuatorianos con edad comprendidas entre 25 y 55 años tiene menor densidad que los precedentes y por tanto es un grupos menos cohesivos.

El Grupo 4 se corresponde más débilmente con los colectivos NC+55pNC y NC25-55pNC, que se aglutinan más con los de origen generacional catalán; globalmente son los de menor cohesión como conjunto. Sin duda, el peso menos denso del colectivo NC25-55pNC pondera a la baja ambos colectivos aglutinados en uno sólo.

Si en la graduación decreciente de las densidades de los grupos se hace una sub-clasificación que agrupa a los grupos 6 y 5, por un lado, y a 3 y 1, por otro, se observa que el criterio que los diferencia es la edad, independientemente del origen de los colectivos, al menos entre los catalanes, con respecto a la densidad de sus colectivos.

El segundo criterio clasificatorio, ya dentro de cada grupo de edad, es el del origen: la densidad es más elevada para los catalanes procedentes de la primera inmigración y sus descendientes que para los colectivos de origen catalán nacidos en Cataluña de padres nacidos en Cataluña.

Los ecuatorianos se aparentan con el Grupo 1 y los marroquíes aparecen dispersos entre los grupos sin una atribución clara a ninguno de ellos.

\section{CONCLUSIONES}

Este artículo ha tratado de articular tres objetivos. El primero se refiere a los conceptos de Cohesión e Integración social que se han estudiado y analizado desde dos aproximaciones diferentes. La primera, denominada Cohesión-Integración relacional se aborda exclusivamente mediante una aproximación reticular, y la segunda, denominada relacional-atributiva, es tratada identificando el resultado precedente, que proviene de indicadores relacionales y por tanto

EMPIRIA. Revista de Metodología de Ciencias Sociales. N. ${ }^{\circ}$ 26, julio-diciembre, 2013, pp. 35-62. ISSN: 1139-5737, DOI: 10.5944/empiria.26.7152 
de estructuras reticulares, por medio de características atributivas. Tal objetivo se ha conjugado metodológicamente con la combinación del análisis de redes socio-métricas y ego-centradas y con la posibilidad de aplicarse tanto a la modalidad de la Cohesión-Integración relacional como la relacional-atributiva. La aplicación y el análisis de Cohesión-Integración se lleva a cabo en colectivos representativos de la estructura social, vinculada a la edad y al origen y a las oleadas migratorias. Son sus atributos respectivos los que caracterizan a los grupos que se generen de las relaciones o redes. La originalidad y la aplicabilidad del artículo estriba en mostrar la posibilidad fáctica de esta doble conjunción, sociométrica frente ego-centrada con grupos estructurados relacionalmente y atributivamente.

El proceso de análisis propuesto ha hecho posible generar estructuras reticulares, esto es, grupos específicos tanto en las redes socio-métricas como en las ego-centradas por criterios exclusivos reticulares configurando así identidades estrictamente reticulares. Por otro lado, dichos grupos han sido también identificados a partir de la naturaleza de los colectivos, teniendo en cuenta la asociación mutua entre grupos y colectivos. Se lleva a cabo así el proceso que va de lo relacional a lo relacional-atributivo y ello, de nuevo, en ambos tipos de redes, de las socio-métricas a las redes ego-centradas.

Es importante también destacar la conjunción y reforzamiento de los dos tipos de diseños concebidos y aplicados. En la primera aplicación, la perspectiva relacional y relacional-atributiva va dirigida a la estructuración de las redes teniendo más en cuenta los criterios de Integración versus formas de segregación o asilamiento, esto es, a las variedades Bridging por el análisis de la red de los Alteri para cada Ego. En este sentido, los Nodos aislados, Diadas y Componentes reflejan desde una posición de máxima ausencia de toda Cohesión a otra, en las Cliques, de Integración. La segunda aplicación relacional-atributiva va más orientada a la Cohesión, más o menos variable por medio de la densidad, o Bonding; es decir como forma de distribución del capital social. El resultado es que desde diferentes aplicaciones y diseños es posible establecer la vinculación entre las micro-estructuras mutuas entre agentes individuales de Alteri con los colectivos provenientes de la estructrua social en los que se insertan los Egos.

Por otro lado, otro de los objetivos, es de carácter más sustantivo, en el sentido de que las aplicaciones y diseños serían reveladoras de la bondad y eficiencia de los métodos y la metodología aplicada, ambos en buena parte proveniente de la teoría y análisis de las redes sociales. Por dos aplicaciones relativamente diferenciadas se ha llegado a conclusiones cuasi similares a la hora de caracterizar atributivamente las estructuras generadas relacionalmente por medio de conceptos vinculados a la idea de capital social, estructurado y distribuido en la Cohesión frente a Integración por los indicadores de Bonding y Bridging.

De manera esquemática se reproduce a continuación, a grandes rasgos, los resultados precedentes. En la primera aproximación se concluye que los colectivos $\mathrm{NC}+55 \mathrm{pNC}$ y NE+55 , personas de más de 55 años, de origen catalán, o español fuera de Catalunya, se caracterizan reticularmente, primero, por la ausencia de dispersión, segmentación y segregación de sus redes; segundo, por una

EMPIRIA. Revista de Metodología de Ciencias Sociales. N. ${ }^{\circ}$ 26, julio-diciembre, 2013, pp. 35-62. ISSN: 1139-5737, DOI: 10.5944/empiria.26.7152 
posición más centrada con relación a la presencia de cliques, o una situación de equilibrio entre Cohesión e Integración. Los colectivos NC25-55pNC y NC25$55 \mathrm{pNE}$, esto es colectivos entre 25 y 55 pero con padres de diferentes procedencias, aunque todos sean catalanes de nacimiento, tienden a mostrar un nivel menor de cohesión. Sus características reticulares son, primero, la de una mayor tendencia a la dispersión, segmentación y segregación de sus redes; segundo, a una menor presencia de cliques, esto es, a una menor articulación de sus grupos internos. Así pues se establece un criterio de edad como generador de cohesión, de un lado y, de otro, de mayor inclinación hacia formas de integración más segregadas o asiladas.

En la segunda aplicación y tomando como criterio la cohesión, por medio de la densidad, aparecen: primero, los colectivos NE +55 y NC+55pNC como más densos, siendo de nuevo la edad de más de 55 años un criterio determinante, independientemente del origen; en segundo lugar, los colectivos NC25-55pNE y en menor medida los NE+55, con origen de nacimiento en España pero fuera de Cataluña, son también más densos que la media; en tercer lugar, los colectivos NC25-55pNC y ecuatorianos, genéricamente más jóvenes; y por fin, el colectivo NC25-55pNC de origen generacional catalán.

\section{BIBLIOGRAFÍA}

ADLER, P. S.; KWON, S. W. (2002): «Social Capital: Prospects for a New Concept», Academy of Management Review, 27(1), pp. 17-40.

AMIN, A. (2002): «Ethnicity and the multicultural city: living with diversity», Environment and Planning, 34, pp. 959-980.

ARNEIL, B. (2006): «The meaning and utility of social capital in liberal democratic politics», en EDWARDS, R.; FRANKLIN, J.; HOLLAND, J. (eds): Assessing Social Capital: Concept, Policy and Practice, Cambridge Scholars Press.

ATRIA, R. (2003): «Capital social: concepto, dimensiones y estrategias para su desarrollo», en ATRIA, R.; SILES, M.; ARRIAGADA, I.; ROBISON, L. J.; WHITEFORD, S.: Capital social y reducción de la pobreza en América Latina y el Caribe: en busca de un nuevo paradigma, Libros de la CEPAL, Santiago de Chile.

BAKER, W.E.; OBSTFELD, D. (1999): «Social capital by design: Structures, strategies, and institutional context», en LEENDERS; GABBAY (eds.): Corporate Social Capital and Liability, Boston: Kluwer, pp. 88-105.

BECK, W.; VAN DER MAESEN, L.; WALKER, A. (1997): «Social quality: from issue to concept», en BECK, W.; VAN DER MAESEN, L.; WALKR, A. (eds) The Social Quality of Europe. The Hague, Kluwer Law International, pp. 263-297.

BEN-TOVIM, G. (2002): «Community cohesion and racial exclusion: a critical review of the Cantle Report»', Renewal 10 (2), pp. 43-48.

BERNARD, P. (1999): Social Cohesion: A Dialectical Critique of a Quasi-Concept?, Ottawa: Strategic Research and Analysis Directorate, Department of Canadian Heritage.

BORGATTI, S.P.; JONES, C. ; EVERETT, M. G.(1998): «Network Measures of Social Capital», Connections 21 (2), pp. 7-36 
BOURDIEU, P. (1986): «The Forms of Capital», en RICHARDS, J.G. (ed.), Handbook of Theory and Research for the Sociology of Education, New York: Greenwood Press, pp. 241-258.

BURT, R. S. (1992): Structural Holes: The Social Structure of Competition, Cambridge, MA: Harvard University Press.

BURT, R. S. (2000): «The Network Structure of Social Capital» Research in Organisational Behaviour, 22, pp. 1-83.

BURT, R. S. (2001): Structural Holes versus Network Closure as Social Capital. En N. LIN, K. COOKY; R. S. BURT (Eds.) Social Capital: Theory and Research. Hawthorn, NY: Aldine de Gruyter, pp. 31-56.

BURT, R. (1997): «The contingent value of social capital». Administrative Science Quarterly, 42 (2) pp. 339-365.

CHEONG, P.; EDWARDS, R.; GOULBOURNE, H.; SOLOMOS, J. (2007): «Immigration, Social Cohesion and Social Capital: A Critical Review», Critical Social Policy, 27, pp. 24-49.

COLEMAN, J. (1988): «Social Capital in the Creation of Human Capital», American Journal of Sociology, 94, pp. 95-120.

ENTZINGER, H., BIEZEVELD, R. (2003): Benchmarking in Immigrant Integration European Commission under contract No. DG JAI-A-2/2002/006, European Research Centre on Migration and Ethnic Relations (ERCOMER).

FESTINGER, L.; SCHACHTER, S.; BACK, K. W. (1950): «Social Pressures in Informal Groups: A Study», Annu. Rev. Sociol., 30, pp. 409-425.

FORREST, R.; KEARNS, A. (2001): »Social cohesion, social capital and the neighbourhood», Urban Studies, 38 (12), pp. 2125-2143.

FUKUYAMA, F. (1995): Trust, the social virtues and the creation of prosperity. London: Penguin Books.

GOUGH, I., OLOFSSON, G. (Eds). (1999): Capitalism and Social Cohesion, Basingstoke: Macmillan.

GRANOVETTER, M. S. (1973): «The strength of weak ties», Am. J. Sociol., 78: pp. 1360-80.

GROSS, N.; MARTIN, W. E. (1952): «On Group Cohesiveness», American Journal of Sociology, 52, pp. 546-54.

GUALDA, E. (2007): Integración Social de los inmigrantes y modelos teóricos que la explican, Universidad de Huelva, España.

JEANNOTTE, M. S. (2003): Social Cohesion: Insights from Canadian ResearchOttawa: Strategic Research and Analysis Directorate, Department of Canadian Heritage.

JENSON, J. (1998): «Mapping Social Cohesion: The State of Canadian Research», Canadian Policy Research Network (CPRN), Study No. Fl03, Ottawa, Canada.

JOHANSON, J. (2001): «The Balance of Corporate Social Capital», en GABBAY, S. M.; LEENDERS, R. A. J. (Ed.), Research in the Sociology of Organizations, Stamford, CT: JAI Press, 8, pp. 1-20.

KEARNS, A.; FORREST, R. (2000): «Social cohesion and multilevel urban governance», Urban Studies, 37 (3-6), pp. 995-1017.

LEVI, M. (1998): «A State of Trust», en BRAITHWAITE, V.; LEVI M. (eds.) Trust \& Governance, New York: Russell Sage Foundation.

LIN, N. (1999): «Building a Network Theory of Social Capital», Connections, 22 (1), pp. 28-51.

LIN, N. (2001): Social Capital: a theory of social structure and action, Cambridge: Cambridge University Press. 
LOPEZ, ROLDÁN, P.; LOZARES, C. (2008b): «La construcción de la muestra. El trabajo de campo de la Encuesta de condiciones de vida y hábitos de la población de Cataluña», Metodologies i Recerques. IERMB, Barcelona, 1, pp. 17-39

LOPEZ-ROLDÁN, P.; LOZARES, C. (2008a): «Implicaciones sociológicas en la construcción de una muestra estratificada», Empiria, 14, pp. 87-108.

LOZARES, C.; LOPEZ-ROLDAN, P.; VERD, J. M.; MARTI, J.; MOLINA J. L., BOLIBAR, M. CRUZ I. (2011): «El análisis de la Cohesión, Vinculación e Integración sociales en las encuestas Ego-net». REDES-Revista hispana para el análisis de redes sociales, 20 (1), disponible en (http://revista-redes.rediris.es).

LOZARES, C.; VERD, J. M. (2011): «De la Homofilia a la Cohesión social y viceversa». REDES-Revista hispana para el análisis de redes sociales, 20 (1), disponible en (http://revista-redes.rediris.es).

LOZARES, C. (1996): «La teoría de redes sociales», Papers, 48, pp. 103-126.

LOZARES, C. (2006): «Valores, Campos y Capitales sociales», Redes. Volumen especial, Bellaterra: Publicaciones UAB, pp. 51-89.

LOZARES, C.; VERD, J. M.; LÓPEZ-ROLDÁN, P.; MARTÍ, J.; MOLINA, J. L. (2011): «Cohesión, Vinculación e Integración sociales en el marco del Capital social», REDES-Revista hispana para el análisis de redes sociales, 20 (1), disponible en (http://revista-redes.rediris.es).

LUHMANN, N. (1988): «Familiarity, confidence, Trust: Problems and Alternatives», en GAMBETTA, D. (Ed.), Trust: Making and Breaking Cooperative relations, Oxford: Basil Blackwell, pp. 94-107.

MOODY, J., WHITE, D. R. (2003): «Social Cohesion and Embeddedness: A hierarchical conception of social groups». American Sociological Review, 68 (1), pp. 103-127.

NAHAPIET, J.; GHOSHAL, S. (1998): «Social Capital, Intellectual Capital and the Organizational Advantage», Academy of Management Review, 23 (2), pp. 242-266.

ONYX, J.; BULLEN, P. (2000): «Measuring social capital in five communities», The Journal of Applied Behavioral Science, 36 (1), pp. 23-42.

O'REILLY, C. A.; ROBERTS, K. H. (1977): «Task Group Structure, Communication and Effectiveness in Three Organizations», Journal of Applied Psychology, 62, pp. 674-81.

PORTES, A. (1998): «Social capital: Its origins and applications in modern sociology», Annual Review of Sociology, 24. pp. 1-24.

PÖYHÖNEN, A. (2005): «Exploring the Dynamic Dimension of Intellectual Capital: Renewal Capability, Knowledge Assets and Production of Sustained Competitive Advantage», A paper presented at the 2005 PMA IC Symposium: Management and Measurement of Intangible Assets and Intellectual Capital: Multidisciplinary Insights. New York, 15-16 December 2005.

PUTNAM, R. (1993): «The prosperous community: social capital and public life», The American Prospect, 13, pp. 35-42.

PUTNAM, R. (1995): «Bowling alone: America's declining social capital», Journal of Democracy, 6 (1), pp. 65-78.

PUTNAM, R. (2001) : «Le déclin du capital social aux Etats-Unis. Entretien avec Robert Putnam», Sciences Humaines, 33, pp. 63.

SCHUSTER, L., SOLOMOS, J. (2004): «Race, immigration and asylum: New Labour's agenda and its consequences», Ethnicities, 4 (2), pp. 267-300.

SZRETER, S.; WOOLCOCK, M. (2004): «Health Social capital, social theory and the political economy of public health». Int J Epidemiol., 33, pp. 650-667. 
WOOLCOCK, M. (2000): «Why should we care about social capital?», Canberra Bulletin of Public Administration, 98, pp. 17-19.

WOOLCOCK, M. (2001): «The Place of Social Capital in Understanding Social and Economic Outcomes», ISUMA, Canadian Journal of Public Policy Research 2 (1), pp. $1-10$.

WOOLCOCK, M.; NARAYAN, N. (2000): «Social Capital: Implications for Development Theory, Research and Policy», The World Bank Research Observer, 15(2), pp. 225-249.

WOOLLEY, F. (1998): «Social Cohesion and Voluntary Activity: Making Connections» Paper presented at the CSLS Conference on the State of Living and the Quality of Life of Canada. Ottawa Canada, October 30-31, 1998. 